\title{
Leitthema
}

Bundesgesundheitsbl 2015 · 58:127-130

DOI 10.1007/s00103-014-2090-5

Online publiziert: 2. Dezember 2014

(c) Die Autor(en) 2014. Dieser Artikel ist auf

Springerlink.com mit Open Access verfügbar

Michael Hummel · Cornelia Rufenach

Institut für Pathologie CBF - Molekulare Diagnostik, Campus Benjamin Franklin, Charité - Universitätsmedizin Berlin, Berlin, Deutschland

\section{Biomaterialbanken als Grundlage für die Entwicklung genetisch basierter Präventionskonzepte}

lungen [1] und Biobanken im Umfeld klinischer Einrichtungen/der Krankenversorgung [2]. In diesen beiden Grundtypen werden nahezu alle im Umgang mit Biobanken relevanten Aspekte unterschiedlich behandelt. Hierauf gehen wir im Folgenden ein.

\section{Epidemiologische oder populationsbezogene Biobanken}

von genetischen Merkmalen, die die Ausprägung einer Krankheit bei noch Gesunden begünstigen können. Die Sammlung, Verwaltung und Bearbeitung von Biomaterialien und der entsprechenden Daten erfolgt zunehmend durch zentralisierte Biobanken, die den hohen Ansprüchen an die Qualität, den Datenschutz, die Sicherheit und Nachhaltigkeit genügen. Kleine Biomaterialbanken wurden häufig im $\mathrm{Zu}$ sammenhang mit einer speziellen krankheitsbezogenen Forschungsfrage aufgebaut. Die neueren Entwicklungen gehen aber hin zu großen, zentralisierten Biomaterialbanken, die eine neutrale Plattform als Serviceeinrichtung darstellen. Sie liefern das „Rohmaterial“ für Forschungsprojekte, ohne mit diesen zu konkurrieren. Dies schließt aber nicht aus, dass diese Biobanken auch selbst Forschung betreiben können (und sollen). Diese Biobankenforschung befasst sich häufig mit der Probenverarbeitung, Probenlagerung und Probenqualität $[1,2]$.

Die Rahmenbedingungen für Biobanken können allerdings je nach Herkunft der Proben sehr unterschiedlich sein. Grundsätzlich lassen sich zwei Typen von Biobanken beschreiben: Biobanken im Kontext von epidemiologischen oder populationsbezogenen Materialsamm-
Die Aktivitäten von epidemiologischen bzw. populationsbasierten Biobanken beziehen sich zumeist auf die Sammlung von Blut- und Urinproben von Probanden aus der Allgemeinbevölkerung in einer bestimmten Region oder in einem definierten Umfeld. Aufgrund der zufälligen Auswahl der Probanden werden in diese Sammlungen überwiegend Proben gesunder Personen eingeschleust, jedoch ist der Einbezug erkrankter Individuen nicht ausgeschlossen. Zusammen mit den Proben werden verschiedene Informationen/Daten (Metadaten) über die Probanden gesammelt. Diese Sammlungen sind sehr langfristig angelegt (prospektive Studien), um später auftretende Krankheiten mit (genetischen) Merkmalen der Probanden korrelieren zu können. Um statistisch abgesicherte Aussagen über Assoziationen zwischen Merkmalen und dem Ausbruch von Krankheiten machen zu können, sind ausreichend große Sammlungen erforderlich. Ziel ist es, über diese Assoziationen genetische Merkmale zu identifizieren, die ursächlich für eine Krankheit sind, um den Krankheitsausbruch oder -verlauf dann präventiv zu beeinflussen. Als Beispiele für derartige Biobanken können die in Deutschland der- zeit anlaufende Nationale Kohorte und im Ausland die britische UK Biobank [3] oder die isländische deCODE Biobank [4] genannt werden. Aber auch regional begrenztere deutsche Sammlungen wie KORA-gen [5] oder PopGen [6] zählen zu dieser Art von Biobanken.

\section{Klinische Biobanken}

Klinische Biobanken verfolgen eine andere Strategie als epidemiologische bzw. populationsbasierte. Bei Probanden im klinischen Kontext handelt es sich in aller Regel um Patienten, die sich aufgrund einer Erkrankung bereits in Behandlung befinden. Entsprechend spiegelt sich hier in den gesammelten Proben direkt der Krankheitszustand wider. Die Bioproben umfassen sowohl Körperflüssigkeiten der Patienten (Blut, Liquor, Urin etc.) als auch Gewebeproben. Zudem liegen aufgrund der Untersuchungen im Klinikumfeld auch umfangreiche medizinische Daten zum Patienten vor, die den Bioproben zugeordnet werden können. Damit lassen sich Erkenntnisse zu krankheitsbezogenen Veränderungen identifizieren, die zur Entwicklung neuer therapeutischer sowie diagnostischer Verfahren und gegebenenfalls auch für die Krankheitsprävention herangezogen werden können. In den letzten Jahren sind in verschiedenen deutschen Forschungseinrichtungen zentrale klinische Biobankstrukturen entstanden, die die vormals verstreuten Biobankaktivitäten vor Ort bündeln, harmonisieren und nachhaltig gestalten sollen. Als Beispiele können hier die BMBF-geförderten zentralisierten Biomaterialbanken (cBMBs) in Aachen [7], Berlin [8], Heidelberg [9], Kiel [10] und Würzburg [11] 
bzw. die sog. $\mathrm{m} 4$ Biobank Allianz [12] in München genannt werden.

\section{Qualität der Biomaterialien}

Unabhängig von der Art der Biobank, ist die Qualität der in ihnen enthaltenen Bioproben und der zugeordneten Daten von zentraler Bedeutung. Retrospektive Untersuchungen belegen, dass selbst in sehr hochrangigen Publikationen die Herkunft, die Qualität und die Zusammensetzung der Biomaterialien häufig nicht oder nur sehr unzureichend beschrieben werden [13]. Diese sehr mangelhafte Ausgangssituation mag dazu beigetragen haben, dass die in den Publikationen beschriebenen Ergebnisse nicht oder nur zum Teil reproduziert werden konnten. Derartige Fehlinterpretationen verursachen bei der pharmazeutischen Industrie jährlich weltweit hohe finanzielle Verluste, da sie häufig Entwicklungen, die auf solchen Publikationen basieren, abbrechen muss. In populationsbezogenen Biobanken können die Qualitätskriterien aufgrund der Art der Probenakquisition von Anfang an deutlich besser implementiert werden als in klinischen Biobanken. Zur Materialakquise im Rahmen populationsbezogener Biobanken werden mit den Probanden Termine vereinbart, sodass die nachfolgenden Prozesse der Material- und Datengewinnung darauf abgestimmt werden können. Im klinischen Kontext hat die Patientenversorgung Vorrang vor der Bioprobengewinnung. Aus diesem Grund lässt sich die Probengewinnung auch nur teilweise zeitlich bestimmen. Auch ist hier der Weg von der Probennahme bis zur Einlagerung in der Regel weiter als in einem speziell zur Probengewinnung ausgelegten Umfeld. Um auch im klinischen Umfeld Proben zu gewinnen, die einheitlichen Qualitätsansprüchen genügen, ist die genaue Erfassung ihres Verlaufs von der Gewinnung bis zur Einlagerung in der Biobank essenziell. Dieser Verlauf wird z. B. im sog. SPREC-Code [14] der Probe dokumentiert.

\section{Rechtliche und ethische Aspekte}

Die Unterschiede zwischen den beiden Grundtypen von Biobanken erstrecken sich auch auf die rechtlichen und ethischen Rahmenbedingungen [15]. In klinischen Biobanken werden die Bioproben meist nicht ausschließlich für ein spezifisches Projekt gesammelt, sondern bieten die Grundlage für die unterschiedlichsten Forschungsschwerpunkte in einer klinischen Einrichtung. Folglich ist zum Zeitpunkt der Probengewinnung nicht immer klar, für welches Projekt und welche Analysen das Material später verwendet werden wird. Daher kann der Patient bei der Probenentnahme häufig nicht in eine bestimmte Verwendung seiner Probe einwilligen, sondern er stimmt zunächst nur deren Lagerung in der Biobank und späteren Verwendung (gemeinsam mit den klinischen Daten) für Forschungszwecke zu. Wenn keine erneute Zustimmung des Patienten zu einem nachfolgenden, spezifischen Forschungsprojekt vorliegt, kann dieses nur nach einem positiven Votum der zuständigen Ethikkommission durchgeführt werden. Damit die klinischen Biobanken in Deutschland hier nun nach vergleichbaren Regeln vorgehen können, hat der Arbeitskreis Medizinischer Ethik-Kommissionen Mustertexte zu unterschiedlichen Aspekten, die in diesem Zusammenhang von Bedeutung sind, entworfen; sie können an die jeweiligen klinischen Verhältnisse angepasst werden [16]. Populationsbasierte Biobanken können hingegen die Patientenaufklärung und -einwilligung projektspezifisch ausrichten und alle ethisch relevanten Aspekte bereits vor Beginn der Sammlung mit der zuständigen Ethikkommission klären. Aus diesem Grund muss bei ihnen zur späteren Verwendung der Proben und Daten kein erneutes Ethikvotum eingeholt werden.

\section{Neue Analysemethoden ermöglichen neue Behandlungskonzepte}

Die Entwicklung neuer Techniken - insbesondere die genomische Hochdurchsatzsequenzierung - eröffnet sowohl den populationsbezogenen als auch den klinischen Biobanken nahezu grenzenlose Möglichkeiten [17]. Die Sequenzierung wird die Identifizierung von tumorassoziierten Veränderungen sowie auch der Zusammenhänge zwischen bestimmten
Krankheiten, genomischen Eigenschaften und demografischen Daten massiv vorantreiben, sodass viele heute noch offene Fragen gelöst werden. Die gewonnenen Antworten werden auch helfen, Personen zu identifizieren, die ein erhöhtes Krankheitsrisiko aufweisen bzw. bei bereits erkrankten Individuen die genomischen Veränderungen zu bestimmen, die z. B. maßgeblich für das Ansprechen auf therapeutische Interventionen (personalisierte Medizin) sind [18].

Komplexen Krankheiten liegen auf genomischer Ebene meist genetische Veränderungen/Variationen in mehreren Genen zugrunde. Genomweite Assoziationsstudien (GWAs) haben diesbezüglich Hunderte von sog. genetischen Markern geliefert. Wenn eine bestimmte Genvariante nicht zufällig mit einer Krankheit auftritt, sondern hier ein statistisch zuverlässiger Zusammenhang besteht, spricht man von einer genetischen Assoziation. Diese legt nahe, dass dieser Marker dann auch an der Krankheitsentstehung beteiligt ist. Allerdings werden bei den immer umfangreicher werdenden GWAs mit immer mehr Proben nur noch mehr genetische Marker mit kleineren Effekten gefunden, sodass Aussagen zu den genetischen Grundlagen einer Krankheit kaum mehr möglich sind. Ziel bei der Erforschung der genetischen Ursachen komplexer Krankheiten muss es daher sein, die für eine Krankheit wesentlichen genetischen Varianten zu identifizieren und ihre funktionelle und pathophysiologische Bedeutung aufzuklären. Dies wird nur möglich sein, wenn die zur Genotypisierung verwendeten Gen-Chips mit anderen sog. Omics-Techniken (Proteomik, Metabolomik, Transkriptomik etc.) und funktionellen Studien kombiniert werden. Hier wird nun die Bedeutung von Biomaterialbanken sehr deutlich, die weitaus mehr bieten als die reine genetische Information.

Diese Entwicklung lässt sich durch die Zusammenarbeit der verschiedenen Biobanktypen nachhaltig vorantreiben. Die kombinierte Auswertung der Proben und Daten aus den populationsbasierten und klinischen Biobanken kann ganz erheblich zur Identifizierung krankheitsrelevanter Gene beitragen.

Die bisherigen Ergebnisse der Sequenzierungen haben auch gezeigt, dass vie- 
le Erkrankungen - insbesondere Tumore - auf molekularer Ebene sehr heterogen sind, obwohl sich auf histologischer und klinischer Ebene keine Unterschiede finden [19]. Das Beispiel des Adenokarzinoms der Lunge mag dies verdeutlichen: Bis vor wenigen Jahren wurden hier nur drei histologische Subtypen unterschieden, heute kennt man mehr als 15 molekulare Subtypen. Diese molekularen Unterschiede sind nicht nur akademisch interessant, sondern auch von großer therapeutischer Bedeutung: Kennt man den molekularen Subtyp des Tumors, können gezielt Substanzen (blockierende Substanzen, Antikörper oder andere „small molecules“) gegen ihn eingesetzt werden, was den therapeutischen Erfolg deutlich verbessert. Zum Auffinden und zur Validierung dieser molekularen Subtypen sind klinische Biobanken von zentraler Bedeutung. Dies gilt nicht nur für das Lungenkarzinom, sondern praktisch für alle Tumorerkrankungen.

\section{Herausforderungen}

Die Unterteilung von Krankheiten in eine immer größere Zahl an molekularen Untergruppen stellt die Biobanken bei der Zusammenstellung großer Kollektive vor erhebliche Probleme. Einerseits müssen die biobankeigenen Proben ausreichend gut molekular charakterisiert sein, um eine geeignete Probenauswahl treffen zu können. Andererseits reichen die eigenen Materialsammlungen oft nicht aus, um große Kollektive mit geeigneten Proben in einem ausreichenden Maße zu generieren. Daher wird es immer wichtiger, dass sich Biobanken vernetzen. Dies setzt - neben der Schaffung der rechtlichen und ethischen Rahmenbedingungen - auch voraus, dass Informationen zwischen den Datenbanken der Biobanken ausgetauscht werden können. Derzeit laufen auf nationaler und europäischer Ebene intensive Vorbereitungen, um diese Voraussetzung für eine Konnektivität von Biobanken zu schaffen. In Deutschland wurde hierfür der Deutsche Biobankknoten eingerichtet (German Biobank Node/BBMRI.de [20]; Leiter ist der Autor dieses Beitrages), der an der Berliner Charité angesiedelt ist und vom $\mathrm{BMBF}$ finanziert wird. Beim Deutschen Biobankknoten laufen die nationa- len Aktivitäten zur Harmonisierung der Qualitätsmanagement- und IT-Konzepte zusammen. Dies erfolgt in enger Kooperation mit dem Deutschen Biobanken-Register (www.biobanken.de), in dem sich die deutschen Biobanken in einer Datenbank präsentieren. Gleichzeitig stellt der Deutsche Biobankknoten das Brückenglied zum europäischen Netzwerk her. Hier gibt es seit 2014 die pan-europäische Biobanken-Infrastruktur BBMRI-ERIC (Biobanking and BioMolecular resources Research Infrastructure (www.bbmri-eric. eu); Leiter ist Jan-Eric Litton), die die einzelnen nationalen Biobankknoten (einschließlich des Deutschen Biobankknoten) vernetzen soll [21].

Die hochdichte Charakterisierung von Bioproben erfolgt nicht nur auf der genomischen Ebene. Auch proteomische, transkriptomische, metabolomische, epigenetische u.a. Daten haben gigantische Ausmaße erreicht, die im Zusammenspiel mit den genomischen Daten nach neuen Lösungen der Datenspeicherung, der Datenverarbeitung und des Datenaustausches rufen. Der Aspekt der Datensicherheit darf in diesem Zusammenhang keinesfalls vernachlässigt werden. Je mehr Daten generiert und ausgetauscht werden, umso größer ist die Gefahr, dass sie auch missbraucht werden könnten. Aus diesem Grund muss alles daran gesetzt werden, die Daten aus Bioproben in einem besonderen Maße zu schützen. Hier setzt das europäische Verbundprojekt BioBankCloud [22] an (Koordinator Jim Dowling, Stockholm), das neben neuen Lösungen zur Speicherung und zum Austausch riesiger Datenmengen auch den Schutz der Daten zum Ziel hat.

\section{Fazit}

Die Bedeutung sowohl der klinischen als auch der populationsbezogenen Biobanken für die Entwicklung neuer diagnostischer und therapeutischer Strategien wird weiterhin stark zunehmen. Die Bereitstellung von Bioproben für die akademische und pharmazeutische Forschung setzt sehr gut charakterisierte Probandenkollektive voraus, was durch neue Hochdurchsatztechnologien ermöglicht wird. Essenziell ist eine kluge Datenvernetzung aller biobankrelevan-
Bundesgesundheitsbl 2015 · 58:127-130

DOI 10.1007/s00103-014-2090-5

(c) Die Autor(en) 2014. Dieser Artikel ist auf Springerlink.com mit Open Access verfügbar

\section{Hummel · C. Rufenach}

\section{Biomaterialbanken als} Grundlage für die Entwicklung genetisch basierter Präventionskonzepte

\section{Zusammenfassung}

Sammlungen von humanen Bioproben und deren Verknüpfung mit zusätzlichen Informationen sind grundsätzlich keine neue Erfindung. Allerdings haben sich in den letzten Jahren die Ansprüche an die Menge, die Qualität und den Informationsgehalt der Bioproben erheblich erhöht. Auch die rechtlichen und ethischen Rahmenbedingungen haben sich entsprechend verändert, ebenso wie der Grad an (IT-)Vernetzung und Kommunikation zwischen Biomaterialbanken. Diese Veränderungen tragen in ihrer Kombination ganz erheblich dazu bei, dass Biomaterialbanken einen zentralen Beitrag zur Entwicklung genetisch basierter Präventionskonzepte leisten.

\section{Schlüsselwörter}

Biobank · Bioproben Prävention .

Personalisierte Medizin .

Gezielte therapeutische Intervention

\section{Biomaterial banks are crucial to developing genetically-based prevention concepts}

\section{Abstract}

Collections of human biological samples and their link to additional information is not a new concept. However, the demands on the quantity, quality, and the information content of biological samples have increased considerably in recent years. The legal and ethical frameworks have changed accordingly, as well as the degree of (IT) networking and communication between biomaterial banks. Due to this development, biobanks contribute significantly to the generation of genetically-based prevention concepts and to new therapeutic approaches.

Keywords

Biobank - Biospecimen Prevention .

Individualized medicine .

Molecular targeted therapy

ten Informationen, um die „Schätze“, die in Deutschland, Europa und der Welt gesammelt werden, auch sinnvoll und ziel- 


\section{führend für Forschungsvorhaben und schließlich auch für die Entwicklung neu- er Präventions- und Behandlungskon- zepte einzusetzen.}

\section{Korrespondenzadresse}

Univ.-Prof. Dr. M. Hummel

Institut für Pathologie CBF - Molekulare

Diagnostik, Campus Benjamin Franklin

Charité - Universitätsmedizin Berlin

Hindenburgdamm 30, 12200 Berlin

michael.hummel@charite.de

\section{Einhaltung ethischer Richtlinien}

Interessenkonflikt. Michael Hummel und Cornelia Rufenach geben an, dass kein Interessenkonflikt besteht.

Open Access. Dieser Artikel unterliegt den Bedingungen der Creative Commons Attribution License. Dadurch sind die Nutzung, Verteilung und Reproduktion erlaubt, sofern der/die Originalautor/en und die Quelle angegeben sind.

\section{Literatur}

1. Ammerlaan W, Trezzi JP, Lescuyer P, Mathay C, Hiller K, Betsou F (2014) Method validation for preparing serum and plasma samples from human blood for downstream proteomic, metabolomic, and circulating nucleic Acid-based applications. Biopreserv Biobank 12(4):269-280

2. Kofanova OA, Fack F, Niclou SP, Betsou F (2013) Combined effect of tissue stabilization and protein extraction methods on phosphoprotein analysis. Biopreserv Biobank 11(3):161-165

3. Zugegriffen: http://www.ukbiobank.ac.uk

4. Zugegriffen: http://www.decode.com

5. Zugegriffen: http://epi.helmholtz-muenchen.de/ kora-gen/index.php?page $=$ Home

6. Zugegriffen: http://www.popgen.de

7. Zugegriffen: http://www.cbmb.rwth-aachen.de

8. Zugegriffen: http://biobank.charite.de

9. Zugegriffen: http://www.klinikum.uni-heidelberg. de/BioMaterialBank-Heidelberg.130088.0.htm

10. Zugegriffen: http://www.uni-kiel.de/medinfo/institut/profil/popgen2/

11. Zugegriffen: http://www.ibdw.ukw.de/aktuell.html

12. Zugegriffen: http://www.m4.de/personalisiertemedizin/m4-biobank-alliance.html

13. Simeon-Dubach D, Perren A (2011) Better provenance for biobank samples. Nature 475, 454-455

14. Betsou F, Lehmann S, Ashton G, Barnes M, Benson EE, Coppola D, DeSouza Y, Eliason J, Glazer B, Guadagni F, Harding K, Horsfall DJ, Kleeberger C, Nanni U, Prasad A, Shea K, Skubitz A, Somiari S, Gunter E (2010) Standard preanalytical coding for biospecimens: defining the sample PREanalytical code. International Society for Biological and Environmental Repositories (ISBER) Working Group on Biospecimen Science. Cancer Epidemiol Biomarkers Prev 19(4):1004-1011
15. Simon JW, Paslack R, Robienski J, Goebel JW, Krawczak M (Hrsg) (2006) Biomaterialbanken: Rechtliche Rahmenbedingungen. Medizinisch Wissenschaftliche Verlagsgesellschaft, Berlin. ISBN: 9783939069140

16. Arbeitskreis der medizinischen Ethikkommissionen. Arbeitsunterlagen/Formulare. http://www.akmed-ethik-komm.de/formulare.html. Zugegriffen: 27. Nov 2014

17. Australian Pancreatic Cancer Genome Initiative, ICGC Breast Cancer Consortium, ICGC MMML-Seq Consortium, ICGC PedBrain, Zucman-Rossi J, Futreal PA, McDermott U, Lichter P, Meyerson M, Grimmond SM, Siebert R, Campo E, Shibata T, Pfister SM, Campbell PJ, Stratton MR (2013) Signatures of mutational processes in human cancer. Nature 500(7463):415-421

18. Dietel $M$, Jöhrens $K$, Laffert $M$, Hummel M, Bläker H, Müller BM, Lehmann A, Denkert C, Heppner FL, Koch A, Sers C, Anagnostopoulos I (2013) Predictive molecular pathology and its role in targeted cancer therapy: a review focussing on clinical relevance. Cancer Gene Ther 20(4):211-221. doi:10.1038/cgt.2013.13

19. Vogelstein B, Papadopoulos N, Velculescu VE, Zhou S, Diaz LA Jr, Kinzler KW (2013) Cancer genome landscapes. Science 339(6127):1546-1558. doi:10.1126/science.1235122

20. German Biobank Node/BBMRI.de www.bbmri.de

21. BBMRI-ERIC http://bbmri-eric.eu

22. http://www.biobankcloud.com 